\title{
La place de l'Église catholique aux Îles-de-la-Madeleine
}

\section{Rose-Delima Gaudet}

Volume 46, 1979

URI : https://id.erudit.org/iderudit/1007118ar

DOI : https://doi.org/10.7202/1007118ar

Aller au sommaire du numéro

Éditeur(s)

Les Éditions Historia Ecclesiæ Catholicæ Canadensis Inc.

ISSN

0318-6172 (imprimé)

1927-7067 (numérique)

Découvrir la revue

Citer cet article

Gaudet, R.-D. (1979). La place de l'Église catholique aux Îles-de-la-Madeleine. Sessions d'étude - Société canadienne d'histoire de l'Église catholique, 46, 99-106. https://doi.org/10.7202/1007118ar

Tous droits réservés @ Les Éditions Historia Ecclesiæ Catholicæ Canadensis Inc., 1979
Ce document est protégé par la loi sur le droit d'auteur. L'utilisation des services d'Érudit (y compris la reproduction) est assujettie à sa politique d'utilisation que vous pouvez consulter en ligne.

https://apropos.erudit.org/fr/usagers/politique-dutilisation/ 


\section{La place de l'Église catholique aux}

\section{Îles-de-la-Madeleine}

Le sujet proposé sera traité d'après des recherches exécutées aux archives du Musée de la Mer et publiées par nous dans un fascicule intitulé Odyssée missionnaire*. Ce n'est qu'une ébauche. mais qui ouvre des horizons sur les activités missionnaires dans des îles qui - encore récemment - n'avaient pas leur place sur la carte géographique du Canada.

\section{LA MISSION}

À quand remonte la première visite des hommes de Dieu dans notre «petit coin de terre»? Les premiers découvreurs, fondatcurs, explorateurs et colonisateurs de notre pays y ont mis pied-à-terre : Cartier, Champlain, Nicolas Denys, François Doublet et autres. Il est vraisemblable qu'il en fut de même des premiers missionnaires de passage à l'époque où seuls les aborigènes y venaient faire la pêche et la chasse. Je laisse à d'autres de pousser cette recherche. Le premier document authentique à ce sujet est le premier registre des baptêmes - mariages - sépultures de Port-Lajoie, - aujourd'hui Charlottetown - poste fondé en 1720, au moment où la France faisait élever dans l'île Royale - aujourd'hui le Cap-Breton - la forteresse de Louisbourg. Le comte de Saint-Pierre en fut le premier scigneur, seigneurie qui comprenait les îles de la Madeleine, qui avaient porté les noms d'îles aux Margots, d'îles Ramćes, d'îles Brion, et même tous ces noms à la fois ... Donc, en 1720, le comte de Saint-Pierre ramena de France où il était de passage un ancien

* Sr Rose-Delima Ginudet, c.n.d., Odysséce missionnairc. Nous nous sommes également inspirée de Cent ans d'éducation aux liles de la Madeleine: Paul Hubert, Les îles de la Madeleine et les Madelinots, Rimouski, 1926. 251 pp. ; Wilfrid PINeAu, ptre, Le clergé français dans l'île du Prince Édouard, Québec, Conseil de Vie française, 1967, 160pp. ; Cyriaque Daiglr: Les anciens missionnaires de l'Acadie, Saint-Louis de Kent, N.B., 1956, 68pp. Nous avons d'autre part consulté la correspondance des premiers missionnaires aux Archives de l'Archevêché de Québec. 
missionnaire de Ville-Marie, le Père Charles-René de Breslay, curé de Notre-Dame, sulpicien, qui y avait vécu au-delà de vingt ans.

Le Père de Breslay mentionne qu'il détient son autorité du consentement de $M^{\star r}$ de Mornay, coadjuteur de l'évêque de Québec. le seul diocèse en Amérique à cette époque.

Le Père de Breslay, aidé du Père Anselme Métivier. sulpicien comme lui, ont visité occasionnellement les pêcheurs des îles de la Madeleine; mais il ne semble pas qu'il y eut des familles résidentes à cette époque. Tout au plus des navigateurs et pêcheurs qui ! avaient pied-à-terre du printemps à l'automne. Il ne semble pas non plus que des missionnaires aient passé l'hiver aux îles durant ce qu'on appelle "la domination française». Depuis 1713. l'Acadie appartenait à l'Angleterre, ce qui durant cinquante ans occasionne des frictions que ne connaît pas le reste du Canada. Le Père de Breslay a eu fort à faire pour défendre les droits de la justice. Persécuté au cours de ses randonnées missionnaires, il doit se réfugier durant quatorze mois dans des campements indiens. L.'Église de Québec le nomma vicaire général en Acadie en 1725. lui conférant ainsi une plus grande autorité.

En l'absence de missionnaire, débute vers cette époque le ministère des Anciens, qui recevront plus tard un mandat officiel de l'évêque de Québec, entre autres celui de Jean Doucet, cité par Cyriaque Daigle dans Les Anciens Missionnaires de l'Acadie. Il fait aussi mention de Pères Capucins et Récollets qui auraient précédé le Père de Breslay; il nomme Isidore Félix et Félix Pain comme étant venus aux îles de la Madeleine.

Les événements funestes de la dispersion des Acadiens et de la guerre de Sept Ans, qui ne se terminent pas avec le traité de Paris en 1763, ont ralenti l'action de l'Église, car les prêtres étaient pourchassés. Des mains des seigneurs français, les îles de la Madeleine sont passées à des seigneurs anglais dont le premier concessionnaire fut Richard Gridley. compagnon de Wolfe aux Plaines d'Abraham en 1759. Il engagea vingt-deux Acadiens pour assurer une chasse et une pêche fructueuses en ces îles qui furent annexées à Terre-Neuve jusqu'en 1774. Ces Acadiens n'acceptèrent le contrat de Gridley qu'à la condition expresse qu'il leur assurerait la présence d'un prêtre au milieu d'eux. C'est ainsi que la première démarche en faveur de l'Église catholique est faite par un non-catholique. Il écrivit à cet effet à l'évêque de Québec qui donna son appui. Mais 
l'Église de Québec ne pouvait se suffire et ne comptait que sur des prêtres européens. On dut attendre plusieurs années, se contentant d'un passage missionnaire occasionnel. Aussi la plupart de ces vingt-deux engagés dont nous connaissons les noms, bien qu'employés aux îles de la Madeleine, laissèrent leurs familles à l'île Saint-Jean jusque vers 1774 , où sous une nouvelle constitution (l'Acte de Québec), ces îles furent annexées au Québec. C'est en cette même année que des Pères du Saint-Esprit offrirent leur ministère en faveur de l'Acadie, à la grands satisfaction des habitants des îles de la Madeleine.

Le premier missionnaire de cette époque fut le Père ThomasFrançois Lcroux, spiritain, qui jusqu'en 1782 parcourut mer et terre. desservant à tour de rôle l'île Saint-Jean, le Cap-Breton, les côtes du Nouveau-Brunswick à Beaubassin, les îles de la Madeleine, étant le seul prêtre accepté par le nouveau régime durant la guerre de l'Indépendance Américaine qui couvre tout son ministère (17751783 ). Sans église, sans demeure, il officiait aux îles dans un pauvre magasin des pêcheurs de Richard Gridley. Voici un fragment de lettre qu'il adressa au grand vicaire Gravé de la Rive :

J'ai eu beaucoup de tribulations. On ma mis le pistolet sur la gorge. J'ai fait naufrage une fois. Je suis tombé à la mer dont j’ai été retiré n'ayant plus ni mouvement. ni connaissance, en revenant de mes missions. Sans compter la faim. la soif, réduit à manger de la soupe à la vache marine. et encore elle était puante. Après tant de travaux. de peines et de fatigues, on cherche à me persécuter mal à propos. Dieu soit béni !

Devenu en 1782 le fondateur de la paroisse de Memrameook. il laissa une grande réputation. Les pêcheurs des îles hivernant à Malpèque dans l'île Saint-Jean, bénéficièrent plus longtemps de ses services religieux.

De 1784 à 1793, le Père William Phelan, capucin, ordonné en Irlande en 1770, vient prendre la cure d'Arichat, dans l'île Madame au Cap-Breton, avec mission des îles de la Madeleine. Les actes des baptêmes, mariages et sépultures de ces années furent pour la plupart détruits dans l'incendie du presbytère d'Arichat en 1838. Aujourd'hui, par les recensements civils, on essaie de récupérer ces actes pour les services de la généalogie. Le missionnaire William Phelan excrça donc durant près de dix ans un ministère itinérant aux îles de la Madeleine. De nouveaux problèmes surgirent, car le régime 
Gridley, - très humain en somme - fut remplacé en 1787 par celui d'Isaac Coffin, un officier de la marine américaine. Ce nouveau concessionnaire se montra d'une exigence telle qu'il provoqua à cette époque un nouvel exode. Immigration et émigration se croisent et persistent durant trois générations : celle de l'oncle, du neveu et du petit-neveu Coffin, qui soumettent leurs sujets à un régime inacceptable. Seul le prêtre peut les défendre, eux ne sachant pour la plupart ni lire ni écrire, et nayant encore aucune organisation civile ni scolaire, étant à la merci du plus fort. Le prêtre se fait à la fois conseiller, avocat, médecin, consolateur, marin, et que sais-je.

\section{LES PAROISSES}

Il arrive dans la vie que le malheur des uns fait le bonheur des autres. En France gronde la Révolution. Des prêtres sont chassés de leur pays. Parmi eux les deux Pères spiritains Jean-Baptiste Allain et François Lejamtel, de Normandie, qu une autorisation de Londres permet de faire passer dans l'immense diocèse de l'Amérique. Transférés de Halifax - où réside le grand vicaire de l'évêque de Québec - aux deux petites îles françaises de St-Pierre et Miquelon, nos deux missionnaires vont à Québec soumettre leurs projets à la haute autorité de $\mathrm{M}^{\mathrm{wr}}$ Hubert et reviennent à St-Pierre et Miquelon travailler à la récupération des débris des familles dispersées et vagabondes depuis 1755 . Le Père Lejamtel se fixera au Cap-Breton, tandis que le Père Jean-Baptiste Allain accompagnera les quarante familles qui débarquent sur le Cap Gridley, devenu le Cap du Musée de la Mer. Ces deux cent cinquante personnes unies aux cent cinquante déjà fixées aux îles de la Madeleine formeront le noyau de la première paroisse ouverte le 28 juillet 1793 sous le vocable de Notre-Dame des Monts, à Havre-Aubert. Le Père Allain fixe certains règlements, fait nommer trois marguilliers : Louis Boudreau. Nicolas Cormier et Joseph Bourgeois, pionniers des Îles, auxquels s'ajoutent bientôt les Arseneau, Bourque, Chiasson, Gaudet, Hébert, Landry. Vigneau, et autres pêcheurs et marins dont les familles se dispersent peu à peu dans les îles et les cantons.

Une première chapelle était déjà sise au pied de la Demoiselle. l'une des collines avoisinantes. Le curé J.B. Allain la fait agrandir sommairement pour y accueillir toutes ses ouailles, qui se plient docilement à ses directives. Il va de nouveau à Québec, en goélette de fortune, exposer les conflits civils du régime Coffin où ni la loi de Terre-Neuve, ni celle de l'Acte de Québec n'avaient été respectées. Ces conflits durèrent plus de cent ans... mais passons. 
La première visite épiscopale aux îles de la Madeleine fut celle de $\mathbf{M}^{\mathrm{rr}}$ Joseph-Octave Plessis, du 19 au 24 juin 1811. Les actes de cette visite sont conservés au registre paroissial de Havre-Aubert. Ils nous fournissent de précieux renseignements sur les habitants des îles et sur leurs rapports avec leur paroisse et avec leur curé. Il y est question d'une nouvelle église qui sera de fait construite en 1813. $\mathrm{M}^{\mathrm{wr}}$ Plessis bénit une croix érigée sur la Demoiselle le dimanche, 23 juin, en souvenir de la première visite épiscopale aux îles de la Madeleine, où enfants et vieillards reçurent le sacrement de confirmation.

Après les Pères Allain et Lejamtel qui se remplacèrent l'un l'autre, à un certain moment, un naufrage fit périr le jeune prêtre Louis Dufresne désigné spécialement par $\mathrm{M}^{\mathrm{ar}}$ Plessis et préparé en conséquence. Ce fut un événement irrémédiable. Les Pères Gabriel Champion, curé de Chéticamp au Cap-Breton, et Jean-Louis Beaubien, curé de Rustico à l'Île-du-Prince-Édouard, se rendent de temps en temps auprès des habitants des îles qui souffrent de la privation des services religieux. Il en a été ainsi jusqu'en 1817 , où le jeune abbé Rémi Gaulin se rend à son tour desservir cette mission escarpée et isolée dans les grandes eaux.

À partir de 1819, M"*r l'Évêque de Québec eut recours à une nouvelle méthode pour assurer un prêtre résident aux îles de la Madeleine : lors de son ordination, le jeune ordonné signe un contrat de trois ans de mission aux îles. Les trois ans écoulés, il est sûr de revenir au pays de Québec. Se sont ainsi succédé aux îles de la Madeleine, demeurant à la mission paroissiale Notre-Dame des Monts sous le vocable de la Visitation : les abbés Jean-Marie Madran (1819-1822), qui passa ensuite en l'Acadie du Nouveau-Brunswick ; Auguste-Magloire Blanchet (1822-1825), qui fit bâtir en 1823 la première chapelle de Havre-aux-Maisons et fut par la suite premier évêque de Nesqually, dans l'Ouest américain ; Pierre Béland (18251827), qui fit terminer la chapelle de Havre-aux-Maisons dépendante de la paroisse mère ; Pierre Bédard (1827-1830), qui assure un premier pied-à-terre missionnaire à l'Étang-du-Nord, petite chapelle bâtie en 1828 ; Pierre-Honoré Brunet (1830-1833), qui fera agrandir cette chapelle, bénite sous le patronage de saint François-Xavier ; Thomas-Léandre Brassard (1833-1836), lui aussi allant de sa paroisse missionner dans les hameaux environnants dispersés le long des côtes ; et L. Noël (1836-1839).

Dans l'intervalle, en 1821, un morcellement de diocèse avait eu lieu par la nomination d'un évêque suffragant auxiliaire de l'Ar- 
chevêque de Québec : $\mathbf{M}^{* r}$ Bernard-Angus MacEachern, qui établit sa résidence à Charlottetown, l'ancien Port-Lajoie. Mr MacEachern mourut en 1835 et fut remplacé par M*r Bernard-Donald MacDonald qui transféra son siège à Rustico, au milieu des Acadiens où il demeura jusqu'à sa mort en 1859 . On remarquera tout de même que les missionnaires des îles de la Madelcine passent toujours par Québec. Il en sera ainsi du jeune abbé A.-Alexis Bélanger. qui ouvre non pas un triennat mais une décennie, car après trois ans, il supplie son évêque de le maintenir à sa paroisse de Havre-Aubert, où il ajoute aux fonctions précédentes celle d'instituteur, ouvrant une première école dans son presbytère en 1839. C'est le début des écoles. Les 1380 ouailles du jeune curé sont avides de connaissances. Il fait venir de son pays natal, Saint-Roch-des-Aulnaies. sa propre sœur, $\mathbf{M}^{\mathrm{m}}$ Bélanger-Morin, première institutrice des îles de la Madeleine, dans une première école bâtie à la paroisse, et par la suite dans les autres îles. Il s'intéresse à l'instruction de jeunes étudiants, dont l'un, Charles-Nazaire Boudreau, deviendra son successeur en 1849 , le premier prêtre natif des îles de la Madeleine : dont un autre, Alexandre Cormier, sera l'initiateur de l'organisation scolaire et civile : municipalité, cours de justice, arrondissement scolaire, enseignement, etc.

Il est beau de voir ce jeune prêtre partager l'exil des émigrants chassés de leur coin de terre par le «règne » de Coffin. Il partira avec un groupe d'entre eux - une vingtaine de familles - vers la baie Saint-Georges, à Terre-Neuve, où il les suit et meurt dans la soixantaine. Les francophones de Terre-Neuve sont les descendants de ces émigrés de 1849.

$\mathrm{M}^{\mathrm{*r}}$ MacDonald, évêque de Charlottetown, voulant soutenir le zèle du curé Bélanger, lui avait obtenu en 1846 un compagnon d'apostolat dans la personne de l'abbé Cajetan Miville-Deschênes, qu'il avait ordonné à Rustico où il résidait toujours. Il fut le premier curé de Havre-aux-Maisons, ce qui sectionna en deux parties le territoire des îles: la première comprend l'île du Havre-Aubert et l'Étang-du-Nord: la seconde comprend le Havre-aux-Maisons et le Cap-aux-Meules.

Il est à propos de souligner le ministère du Père Charles-Nazaire Boudreau de 1849 à 1888 . Il poursuit l'œuvre commencée par le curé Bélanger, fait face aux mêmes problèmes de l'émigration, se voit de nouveau seul curé des îles durant deux ans, bâtit un couvent- 
presbytère pour y abriter des religieuses en vue de la formation de maîtresses d'écoles, et que sais-je ...

En 1872, il voit à la construction d'une église au Bassin et y assure un ministère en même temps qu'à sa paroisse de Havre-Aubert et qu'à la chapelle de l'Étang-du-Nord, jusqu'en 1877, à l'arrivée d'un premier prêtre au Bassin, Félix Van Blerk, un Belge en service à l'Île-du-Prince-Édouard. Il accepte la décision épiscopale qui place les religieuses arrivées en 1877 sous la vigilance du nouveau curé de Havre-aux-Maisons, M. Onésime Hébert, devenu plus tard sulpicien.

Cette date, 1877, est mémorable à cause de l'arrivée aux Îles des Sœurs de la Congrégation de Notre-Dame qui ouvrent un pensionnat pour l'éducation des filles et la préparation de maitresses d'écoles. C'était depuis 1854 le rêve des deux chefs que nous avons vus à l'œuvre: Charles-Nazaire Boudreau et Alexandre Cormier. Ils le voyaient enfin réalisé.

Qu'en est-il de la chapelle de l'Étang-du-Nord? Un missionnaire de l'Ouest canadien, natif d'Yamaska, avait séjourné dans les missions d'Acadie. Il fut nommé pour succéder au deuxième curé de Havre-aux-Maisons, M. Azade Trudel. Il s'agit du Père Georges Belcourt dont la vie n'est pas assez connue bien qu'écrite depuis plus de vingt ans. C'est lui qui entreprend la construction d'une église, non plus à l'Étang-du-Nord où était la chapelle, mais à Lavernière, hameau plus central. C'est aujourd'hui la partie transversale formant le transept de l'église actuelle et dont la façade était tournée vers l'Étang-du-Nord. On rapporte que le Père Belcourt, après sa messe très matinale au Havre-aux-Maisons, parcourait à pied la distance jusqu'à Lavernière pour y suivre les travaux et y mettre la main. Rien de surprenant s'il succomba à la tâche en 1874 . Cette petite église ouverte au culte occasionnel ne recevra son premier curé qu'en 1879, M. Théophile Allard.

Voilà donc une Église en pleine effervescence répandue sur tout l'Archipel; car à Grande-Entrée où la plupart des familles sont catholiques, lc curé Edmond Meunier fait bâtir en 1886 l'église actuelle, rénovée depuis. Elle dépendra de Havre-aux-Maisons jusqu'à l'arrivée de son premier curé, M. Jean Buote, de l'Île-du-PrinceÉdouard, en 1928. Quant à la Pointe-au-Loup, son école-chapelle sera remplacée par l'église actuelle au début des années '60.

Depuis 1821, les îles de la Madeleine font partie du diocèse de Charlottetown. Les prêtres acadiens sont pour la plupart acheminés vers les îles de la Madeleine ou transférés dans les missions 
du Nord-Ouest, alors que les paroisses françaises de l'Île-du-PrinceÉdouard sont desservies par des curés de langue anglaise. C'est une anomalie. M $M^{a r}$ Albini Leblanc, évêque de Gaspé, en faisant passer les îles de la Madeleine dans son diocèse, en 1946, corrige en même temps cette situation pénible pour les insulaires, nos voisins. Il est vrai aussi que notre appartenance politique, civile et scolaire appelait cette nouvelle allégeance ecclésiale, mais la brisure n'en fut pas moins profondément ressentie.

Le Congrès des vocations, du 5 au 8 août 1948, la subdivision de la populeuse paroisse de Saint-Pierre de Lavernière par la fondation de celle de Notre-Dame du Rosaire de Fatima, sont des actes posés par $\mathrm{M}^{\mathrm{ar}}$ Albini Leblanc. En 1960, une troisième subdivision de cette même paroisse crée celle de Cap-aux-Meules sous le patronage de Saint-André. Actuellement, les sept paroisses, la mission de Pointe-au-Loup et les œuvres catholiques régionales sont administrées par douze prêtres dont plusieurs courbent sous le poids des ans.

Des communautés religieuses assurent certains services dans des secteurs précis: les Sœurs de la Charité de Québec, fondatrices de l'hôpital Notre-Dame de la Garde en 1938, sont encore présentes au Centre Hospitalier de l'Archipel. Les Frères du Sacré-Cœur, qui œuvrent dans l'éducation depuis vingt-cinq ans, assurent en plus l'œuvre de pastorale de l'École polyvalente par l'ordination sacerdotale du Frère Julien Bonneau. Des Oblates de Marie-Immaculée ont rendu de précieux services aux îles dans les secteurs enseignants et hospitaliers. Les Filles de Marie de l'Assomption, de Campbellton, ont œuvré douze ans ici dans l'enseignement. Les Sœurs de la Congrégation de Notre-Dame y demeurent depuis cent deux ans.

La place actuclle de l'Église catholique aux îles de la Madeleine révèle une espérance en l'avenir : un futur ordonné chez les Pères Trinitaires; deux étudiants en théologie en vue du sacerdoce chez les Rédemptoristes; une probaniste chez les Sœurs de la Charité de Québec. Si l'on ajoute à cela le mouvement de Service de Préparation à la Vie et le mouvement charismatique de prières hebdomadaires, on peut parler d'un avenir plein de promesse.

Sr Rose-Delima Gaudet, C.N.D., Îles-de-la-Madeleine. 